\title{
Prediabetes and subsequent pancreatic fat accumulation
}

\author{
Tomoyuki Kawada ${ }^{1}$ (D)
}

Received: 26 March 2018/ Accepted: 26 April 2018/Published online: 12 May 2018

(C) Japanese Society of Gastroenterology 2018

Yamazaki et al. [1] investigated the effect of prediabetes on subsequent pancreatic fat accumulation, based on the hypothesis that pancreatic fat was a manifestation of disturbed glucose metabolism. Prediabetes was defined as fasting plasma glucose of $100-125 \mathrm{mg} / \mathrm{dl}$ or hemoglobin A1c of $5.7-6.4 \%$, and the change of pancreatic fat was evaluated by computed tomography (CT). A total of 198 nondiabetic participants were composed of 48 prediabetes and 150 non-prediabetes participants. By multiple linear regression analysis, baseline prediabetes was associated with future pancreatic fat accumulation with beta value (95\% confidence interval) of 3.14 (1.25-5.03). In addition, body mass index (BMI) and impaired fasting glucose (IFG) were also risk factors of pancreatic fat accumulation. I have some queries on their study.

First, the authors used prediabetes or IFG as an independent variable for the change of pancreatic fat accumulation, by adjusting several variables. As impaired glucose tolerance (IGT) value could not be used as an independent variable, the lack of IGT information for the definition of prediabetes should be specified by further study [2].

Second, BMI was selected as a significant independent variable for the change of pancreatic fat accumulation. I suppose that the amount of visceral fat at baseline by CT

This comment refers to the article available at https://doi.org/10.1007/ s00535-017-1422-2.

Reply to this letter is available at https://doi.org/10.1007/s00535-0181475-x.

\section{Tomoyuki Kawada}

kawada@nms.ac.jp

1 Department of Hygiene and Public Health, Nippon Medical School, 1-1-5 Sendagi, Bunkyo-Ku, Tokyo 113-8602, Japan could also be used as an independent variable. Although liver fat did not become a predictor, visceral fat as another obesity indicator should be checked for the analysis [3].

Finally, the authors selected multiple linear regression analysis. I think that the authors could use prediabetes indictors at baseline as continuous variables. In addition, the change of prediabetes information can be used in combination with the change of pancreatic fat accumulation. Anyway, further studies are needed to know the causal association to confirm the hypothesis that pancreatic fat is a manifestation of disturbed glucose metabolism.

\section{Compliance with ethical standards}

Conflict of interest The author declares that he has no conflict of interest.

\section{References}

1. Yamazaki H, Tauchi S, Kimachi M, et al. Independent association between prediabetes and future pancreatic fat accumulation: a 5-year Japanese cohort study. J Gastroenterol. 2017. https://doi. org/10.1007/s00535-017-1422-2.

2. Huang Y, Cai X, Mai W, et al. Association between prediabetes and risk of cardiovascular disease and all-cause mortality: systematic review and meta-analysis. BMJ. 2016;355:i5953.

3. Graffy PM, Pickhardt PJ. Quantification of hepatic and visceral fat by $\mathrm{CT}$ and MR imaging: relevance to the obesity epidemic, metabolic syndrome and NAFLD. Br J Radiol. 2016;89:20151024. 